\title{
Tuberculosis Infection Control Project Management Experience and Its Application in COVID-19 Response
}

\author{
Qingyun Sun'; Xiaofeng Yan ${ }^{1, * *}$; Cheng Chen ${ }^{1}$; Xuemei Jiang ${ }^{1}$; Jun Cheng ${ }^{2, *}$
}

The outbreak of COVID-19 resulted in many infections, including patients in health care settings (1). Although the main mode of transmission of COVID-19 is by droplets, both tuberculosis (TB) and COVID-19 are respiratory infections that can be spread by airborne transmission. Therefore, a set of systematic and comprehensive nosocomial infection control policies is necessary to control infectious sources, block transmission, and protect people at risk in health care settings.

Surveys showed that the TB infection prevention and control (IPC) status in health care centers were poor resulted from absence of policy support, unreasonable environmental layout, insufficiency of IPC knowledge, shortage of protective appliances (2). Elevated capacity and improved practice, profiting from a systematic training and intervention, would be very useful for better IPC in facilities. In order to improve IPC work based on the strengthened knowledge and skill of staff, a set of IPC activities were designed and conducted in a collaborated project named Building and Strengthening Infections Control Strategies for TB (TB BASICS), developed by China CDC and US CDC. Chongqing Public Health Medical Center (CPHMC), being an infectious disease and public health emergency designated hospital at provincial level, took the responsibility to cooperate with other hospitals during the implementation of this project. TB BASICS has been carried out from July 2018 to June 2019.

Since the outbreak of COVID-19, CPHMC improved its COVID-19 epidemic response by using the experience and lessons learned from implementing this infection control project, to reach the goal of zero infections among healthcare staff who were battling COVID-19 in frontline.

\section{TB BASICS PROJECT IMPLEMENTATION}

TB IPC measures are categorized into three hierarchical groups of measures: administrative, environmental, and personal protection (3-4). The facility-wide administrative measures focus on activities and policies that will support the implementation of systematic IPC in the entire facility. The other administrative measures focus on reducing the risk of exposure by implementing systems and policies to quickly detect, separate, and effectively treat TB cases as well as respiratory hygiene. Environmental measures, such as sufficient ventilation and germicidal ultraviolet, focus on reducing the concentration of $M$. tuberculosis droplet nuclei and prevent their spread. Personal protection measures, mainly using respirator, serve as a complement to the previous two measures to further reduce the risk of exposure of healthcare workers to $M$. tuberculosis $(3,5)$.

TB BASICS is a continuous quality improvement project that aims to implement and strengthen sustainable TB IPC strategies in health facilities through continuous practice, evaluation, and improvement. The CPHMC initiated TB-BASICS project in July 2018, covering 16 areas of the facility deemed to be at risk for TB transmission, including the infection control unit, tuberculosis clinics, in-patient wards, laboratories, and other specialized departments. To realize continuous improvements of $\mathrm{TB}$ IPC measures in all parts, the CPMHC conducted baseline assessments, established intervention control teams, and developed a TB IPC improvement plan and standard operation procedure (SOP) during a 12month time period.

\section{RESULTS AND REFLECTIONS OF THE PROJECT}

Evidence points to TB BASICS having improved our expertise in and attention to IPC. First, support from the facility leadership guaranteed the successful implementation and improvement of TB IPC. Implementing TB BASICS elevated leadership's understanding and support for TB IPC including supporting an investigation of the status of $\mathrm{TB}$ 
infection and disease among health care workers (HCWs) in CPHMC since 2013, strengthening the supply of disinfection equipment, improving patients' pathway, and increasing financial support, and providing disposable surgical masks free of charge for all ambulatory and hospitalized TB patients. Second, hospital layouts and patient diagnosis procedures have been improved, such as better ventilation in sputum collection rooms, an online appointment registration system was developed that assigns patients to consultation times in order to reduce waiting times and waiting room crowding. Third, increased awareness of TB IPC among HCWs has led many of them to take the initiative to improve their daily practice of diagnosis and treatment through multiple methods, especially training (5-6), with increased correct rate from $40 \%$ at baseline to $70 \%$ at final evaluation. Fourth, daily material and equipment meetings are held to discuss the availability of personal protective equipment (PPE), in particular respirators, which give priority to frontline HCWs in isolation areas. Furthermore, HCWs became more attentive to personal protection. It has been observed that all staff starting work on the isolation ward actively ask for respirator fit-testing and use the respirators properly at work.

\section{IMPACT OF TB BASICS PROJECT ON COVID-19 RESPONSE}

CPHMC was designated as a COVID-19 hospital in Chongqing on January 21, 2020, and diagnosed and treated its first confirmed COVID-19 patient on January 24, 2020. Up to March 15, 2020, 224 patients (14 critical, and 17 severe), have been admitted. All patients were admitted to three isolation buildings with six wards (including one negative pressure ward) and a total of $430 \mathrm{HCWs}$ from 61 departments of CPHMC worked in these isolated areas.

The participation of TB BASICS project laid a solid foundation for us to manage this challenge. COVID19 is a respiratory infectious disease like tuberculosis. Tuberculosis is mainly airborne, but COVID-19 is mainly spread by droplets and contact. Based on the current view point, both of them can be spread by aerosols. The management of COVID-19 response was enhanced by the lessons learned from TB BASICS. Scientific and rational COVID-19 response strategy was established and more than 20 standardized workflows were developed including access to the
COVID-19 isolation area, patient transfer, disinfection of emergency vehicles, donning and doffing of PPE, among others, which covered administrative control, environmental control and personal protection equipment. Several IPC guidelines were compiled to guide the epidemic response and printed for easy access by facility staff.

After receiving the task of treating COVID-19 patients, the CPHMC, based on its experience learnt in TB BASCIS, offered personnel and financial support, established COVID-19 Emergency Team. All staff of the infection control department engaged in workflow development. The function of each room and pathway of HCWs were discussed thoroughly and the best way was determined to avoid cross contamination. Some physical barriers were built for protecting HCWs from infection. In addition, in order to make all staff familiar with new workflow, training and drills were carried out, and further revisions were made to improve feasibility. After several rounds of drills, an optimized pathway was agreed upon which guaranteed smooth workflow and reduced transmission risk for all HCWs.

Since January 20, 2020, more than 20 intensified IPC trainings and workflow rehearsals were conducted. Before participating in the treatment of patients with COVID-19, all HCWs must receive IPC training and pass the assessment before entering the isolation ward. These training courses were based in part on information learned from TB BASICS, covered IPC guidelines and best practices, and helped HCWs comply strictly with the requirements that aim to reduce the risk of COVID-19 transmission. Trainings on standard prevention measures based on transmission mode $(G)$ were strengthened.

Using principles and techniques learned from $\mathrm{TB}$ BASICS, environmental measures in isolation area were improved. Before COVID-19 patients were moved into isolation wards, a transmission risk assessment was performed in each area. The air changes per hour $(\mathrm{ACH})$ were measured to assess the ventilation with the goal of achieving $12 \mathrm{ACH}$ in all isolation areas. For areas with poor ventilation (did not meet $12 \mathrm{ACH}$ criteria), upper room GUV fixtures were installed to supplement poor ventilation. One hundred sets of upper-room GUV fixtures were purchased for replenishment, and a robotic disinfection machine and other disinfection facilities were acquired and used. HCWs were assigned to sterilize the isolation area every day at least twice. Work in isolation area being completed, infection control department has taken 
samples from the isolation area. And bacterial cultures of 200 samples were all negative.

In personal protection, N95 respirator fit-testing was provided. The respirators for all staff were selected based on fit testing results. Every staff who was assigned to the isolation area was given one-on-one training on respirator use to guarantee their correct use of the respirator without leakage. A total of 428 staff were given fit-testing to make sure that each staff wore his/her own respirator suitably (7). Moreover, the CPHMC provided surgical masks for all COVID-19 patients without charge.

After completing all work in the isolation area, all HCWs tested negative for COVID-19 using RT-PCR tests for SARS-CoV-2. In addition, after a 14-day quarantine, all HCWs had 2-3 follow up specimens and tested by RT-PCR for SARS-CoV-2, all of which were negative. However, a small investigation of PPE outer surfaces including 5 sets of randomly selected goggles and respirators using environmental sampling methods illustrated detection of SARS-CoV-2 on 1 of the five sampled sets. These results and the fact that none of our HCWs were infected with SARS-CoV-2 highlights the protective effectiveness and proper doffing of PPE among our HCWs.

\section{CONCLUSION}

Benefiting from the establishment of new IPC concepts and technical measures introduced by $\mathrm{TB}$ BASICS, the IPC measures in CPHMC were systematically improved as was the knowledge, awareness, and practices of IPC among HCWs. The capacity enhancement through TB BASICS project has laid a solid foundation for us to make correct strategies for fighting COVID-19, and to successfully complete the various IPC tasks, achieving the goal of "zero infection" for HCWs. The IPC team won the highest trust of all staff, and was known as the "guardian" by the HCWs.
Acknowledgement: We appreciate technical input for TB BASICS project implementation provided from Paul A. Jensen, Sarah E. Smith-Jeffcoat, Michele L. Pearson, RJ Simonds, and Ling Hao from US CDC and Canyou Zhang and Hui Chen from China CDC. We appreciate manuscript editing by Sarah E. SmithJeffcoat and RJ Simonds.

Funding: The TB BASICS project was funded by the China-U.S. collaboration on TB control cooperative agreement.

doi: $10.46234 / \mathrm{ccdcw} 2020.183$

\# Corresponding authors: Xiaofeng Yan, 2429918342@qq.com; Jun Cheng, chengjun@chinacdc.cn.

\begin{abstract}
${ }^{1}$ Department of Nosocomial Infection Control and Prevention, Chong Qing Public Health Medical Center, Chong Qing, China; ${ }^{2}$ National Center for Tuberculosis Control and Prevention, Chinese Center for Disease Control and Prevention, Beijing, China.
\end{abstract}

Submitted: July 18, 2020; Accepted: August 14, 2020

\section{REFERENCES}

1. Huang CL, Wang YM, Li XW, Ren LL, Zhao JP, Hu Y, et al. Clinical features of patients infected with 2019 novel coronavirus in Wuhan, China. Lancet 2020;395(10223):497 - 506. http://dx.doi.org/10.1016/ S0140-6736(20)30183-5.

2. Zhao F, Cheng J, Cheng SM, Zhang H, Zhao YL, Zhang CY, et al. The current status and challenges regarding tuberculosis infection control in health care facilities in China. Biomed Environ Sci 2015;28(11):848 54. http://dx.doi.org/10.1016/S0895-3988(15)30117-3.

3. World Health Organization. WHO guidelines on tuberculosis infection prevention and control 2019 update. Geneva: World Health Organization; 2019. http://www.doc88.com/p-9807872551083.html.

4. World Health Organization. Guidelines on core components of infection prevention and control programmes at the national and acute health care facility level. Geneva: World Health Organization; 2016. https://www. who.int/gpsc/ipc-components/en/.[2020-7-18].

5. Gizaw GD, Alemu ZQ, Kibret KT. Assessment of knowledge and practice of health workers towards tuberculosis infection control and associated factors in public health facilities of Addis Ababa, Ethiopia: a cross-sectional study. Arch Public Health 2015;73(1):15. http://dx.doi.org/10.1186/s13690-015-0062-3.

6. Mah MW, Deshpande S, Rothschild ML. Social marketing: a behavior change technology for infection control. Am J Infect Control 2006; 34(7):452 - 7. http://dx.doi.org/10.1016/j.ajic.2005.12.015.

7. Wang LX, Cheng SM, He GX, Chen MT. China manual of tuberculosis prevention and control. Beijing: China Union Medical University Press. 2010;p.8-9,33. (In Chinese). 